\title{
Point spread function reconstruction simulations for laser guide star multi- conjugate adaptive optics on extremely large telescopes
}

L. Gilles, L. Wang, C. Boyer

L. Gilles, L. Wang, C. Boyer, "Point spread function reconstruction simulations for laser guide star multi-conjugate adaptive optics on extremely large telescopes," Proc. SPIE 10703, Adaptive Optics Systems VI, 1070349 (18 July 2018); doi: $10.1117 / 12.2315793$

Event: SPIE Astronomical Telescopes + Instrumentation, 2018, Austin, Texas, United States 


\title{
Point spread function reconstruction simulations for laser guide star multi-conjugate adaptive optics on extremely large telescopes
}

\author{
L. Gilles ${ }^{1 \mathrm{a}}$, L. Wang ${ }^{\mathrm{a}}$, C. Boyer ${ }^{\mathrm{a}}$ \\ ${ }^{a}$ Thirty Meter Telescope International Observatory LLC, 100 W. Walnut St, Pasadena, CA 91124, \\ USA;
}

\begin{abstract}
This paper discusses point spread function reconstruction (PSFR) simulations for laser guide star (LGS) multi-conjugate adaptive optics (MCAO) on extremely large telescopes (ELTs). The Multithreaded Adaptive Optics Simulator (MAOS), configured to simulate the Thirty Meter Telescope (TMT) Narrow Field InfraRed Adaptive Optics System (NFIRAOS), provided simulated telemetry. For median Mauna Kea turbulence conditions ( 0.55 arcsec seeing) and for the expected NFRIAOS laser guide star (LGS) wavefront sensor (WFS) measurement noise, the actuator error based PSFR algorithm leads to Strehl Ratio (SR), enclosed energy (EE) and PSF profile errors below $8 \%$ in Z-band, $5 \%$ in J-band and $2 \%$ in Kband assuming perfect knowledge of the turbulence and wind profiles and of measurement noise. Further algorithm optimization could in principle reduce this residual error level.
\end{abstract}

Keywords: Laser guide star multi-conjugate adaptive optics (LGS MCAO), point spread function reconstruction (PSFR)

\section{INTRODUCTION}

Point spread function reconstruction (PSFR) has gained increased attention in the adaptive optics (AO) community with the design and construction of extremely large telescopes (ELTs) [1]-[8]. In this paper, laser guide star (LGS) multi-conjugate adaptive optics (MCAO) PSFR simulation results are presented for the Thirty Meter Telescope (TMT) first-light LGS MCAO system, NFIRAOS. An overview of NFIRAOS is provided in [9]. The PSFR algorithm uses the following system telemetry data from the MCAO real time controller (RTC): actuator error covariance matrix (includes both high-order (HO) and low-order (LO) modes, MCAO control matrix (HO and LO), deformable mirror (DM) poke matrix, measurement noise covariance matrix for each HO and LO wavefront sensor (WFS). Knowledge of the turbulence and wind profiles is required. The former can be obtained to high accuracy (1-2\% error) using e.g. a slope detection and ranging (SLODAR) algorithm [10]. Accurate models of aliasing and generalized fitting play an essential role in addition to RTC telemetry data. A schematic block diagram of the PSFR algorithm is provided in Figure 1. The long-exposure optical transfer function (OTF) (Fourier transform of the long-exposure PSF) is obtained as a product of four OTFs: generalized fitting OTF (standard fitting and projection error), aliasing OTF, de-noised actuator error OTF (HO and LO), and static aberration OTF.

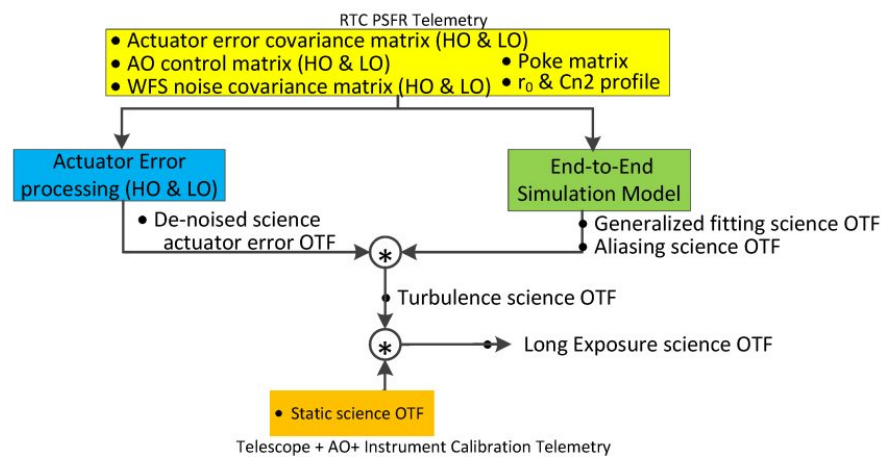

Figure 1: Block diagram of the PSFR algorithm.

${ }^{1}$ lgilles@caltech.edu; phone 1-626-395-1622

Adaptive Optics Systems VI, edited by Laird M. Close, Laura Schreiber,

Dirk Schmidt, Proc. of SPIE Vol. 10703, 1070349 - @ 2018 SPIE

CCC code: $0277-786 X / 18 / \$ 18 \cdot$ doi: $10.1117 / 12.2315793$ 
The actuator error based PSFR algorithm 1 in [1] is the algorithm of choice since it can naturally be configured for any AO mode (classical NGS AO, LGS AO, and MCAO). The following points are worth noting:

- It is desirable to up-sample the actuator influence functions to compute $H_{a, \mathrm{te}}$ in Eq.(2.4) of [1], so as to map the actuator grid onto a finer aperture-plane grid prior to the computation of the long-exposure optical transfer function (OTF) from the actuator error and actuator noise covariance matrices. Up-sampling by a factor of 2 appears to be sufficient.

- For LGS MCAO, the aliasing OTF, $K_{\text {sca }}$ in Eq.(2.3) of [1], is computed for guide stars at finite range. This filter is pre-computed using closed-loop simulations and is averaged over multiple turbulence realizations.

\section{SIMULATION RESULTS}

\subsection{Geometry parameters}

All simulations were performed using the Multi-threaded adaptive optics simulator (MAOS) [10] using a physical optics LGS WFS model, and the default NFIRAOS parameters [11]. The main simulation parameters are: TMT segmented aperture, 7-layer median Mauna Kea turbulence profile which is characterized by $r_{0}=18.6 \mathrm{~cm}, \theta_{0}=2.3 \operatorname{arcsec}, \theta_{2}=8.9 \operatorname{arcsec}$, $\mathrm{f}_{\mathrm{G}}=21 \mathrm{~Hz}$ at $500 \mathrm{~nm}, 6$ high-order guide stars $(5$ on a 70 arcsec diameter circle plus 1 on-axis), order 60x60 ShackHartman LGS wavefront sensing at $800 \mathrm{~Hz}$ using a constrained matched filter, 1 tip/tilt/focus (TTF) and 1 tip/tilt (TT) natural guide stars (NGSs) forming an equilateral triangle of $20 \mathrm{arcsec}$ width centered on-axis, one deformable mirror $(\mathrm{DM})$ of order $63 \times 63$ conjugate to ground, a second $\mathrm{DM}$ of order $76 \times 76$ conjugate to $11.8 \mathrm{~km}, 30 \%$ inter-actuator coupling (IAC), Nyquist sampled science PSFs (Nyquist sampling in J-band is $\lambda_{J} /(2 D) \simeq 4.3$ mas) sampled within a $34 \mathrm{arcsec}$ wide field of view (FoV) centered on-axis, all wavefronts computed with $1 / 64 \mathrm{~m}$ sampling. The 7,000 x 32,000 MCAO minimum variance control matrix is pre-computed offline using 100 iterations of the Fourier domain preconditioned conjugate gradient algorithm for the tomography component and Cholesly back solves for the DM fitting component [11]. Five LO modes (tip/tilt and 3 plate-scale modes) are controlled separately from the HO modes using the split atmospheric tomography architecture [12]. 20,000 time steps (25sec of simulated integration time) with $256 \mathrm{~m}$ wide translating atmospheric phase screens with $1 / 64 \mathrm{~m}$ sampling were simulated, and a total of 10 simulations were performed, each for a different atmospheric turbulence realization.

WFS noise was simulated at the level of 900 photo detected electrons (PDEs) per subaperture per frame for the HO LGS WFSs (spot elongation was simulated, LGS WFSs focused at 90km range), and 2mas root mean square (RMS) per subaperture per frame for the LO TTF and TT NGS WFSs. Since the simulated NGS asterism is symmetrical with respect to FoV and centered on-axis, the science RMS WFE is also symmetrical with respect to FoV. It is therefore sufficient to analyze PSFR performance at 3 field points: on-axis, at a mid-vertex field point (17 arcsec off-axis) and at a corner field point (24arcsec off-axis)

\subsection{Perfect knowledge of atmospheric turbulence profile}

Performance results in terms of long exposure (LE) Strehl Ratio (SR) are plotted in Figure 2. The different error terms entering the atmospheric turbulence part of the PSFR algorithm are displayed. Those terms are: (i) the denoised actuator error OTF (incorporating both HO and LO contributions), (ii) the aliasing OTF, and (iii) the generalized fitting OTF. It can be seen that the PSFR algorithm currently underestimates SR. More accurate modelling of the different error terms, and in particular of the aliasing term, which is assumed to be field-independent in the current model, could in principle reduce this error. Figure 2, right panel, indicates that the science Strehl Ratio (SR) for an off-axis field point slightly varies across different simulated atmospheric turbulence realizations. Hence, for completeness, PSFR accuracy has been evaluated over 10 different realizations. Figure 3 displays PSFR accuracy (see [1] for a definition of the performance metrics) versus field. For the LE science PSF averaged over the 10 realizations of atmospheric turbulence, all performance metrics are below $8 \%$ in Z-band, below 5\% in J-band, and below $2 \%$ in K-band. Figure 4 displays enclosed energy (EE) and PSF profile errors as a function of integration window size, illustrating that the PSF profile error has converged once the integration window reaches a few tens of Nyquist sampled pixels. Figure 5 displays modulation transfer function (MTF) and MTF estimation error cross-sections. 

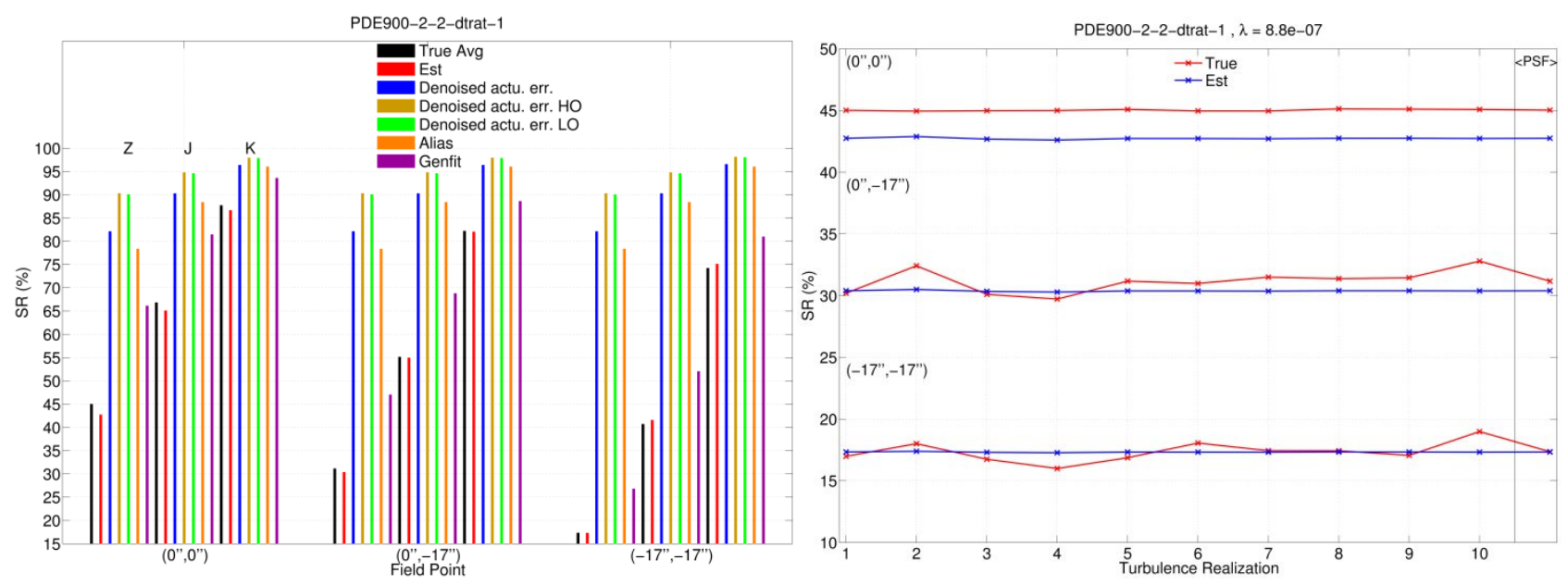

Figure 2: Left: LE SR versus field. Right: Z-band SR (true and estimate) versus simulated turbulence realization.
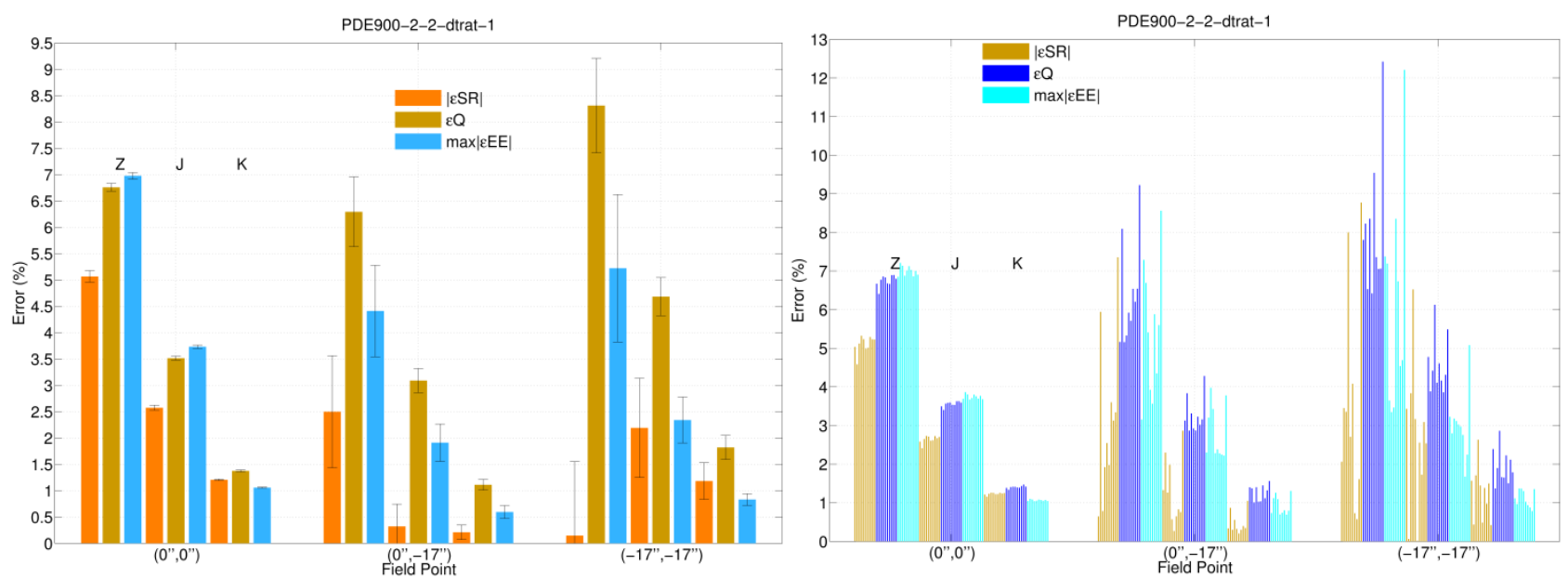

Figure 3: Left: LE SR error ( $\epsilon \mathrm{SR})$, PSF profile error $(\epsilon \mathrm{Q})$ and maximum EE error $(\epsilon \mathrm{EE})$ versus field. Right: SR, PSF profile and maximum encircle energy error versus field and turbulence realization (each set of 10 identically colored bars is for the 10 atmospheric turbulence realizations).

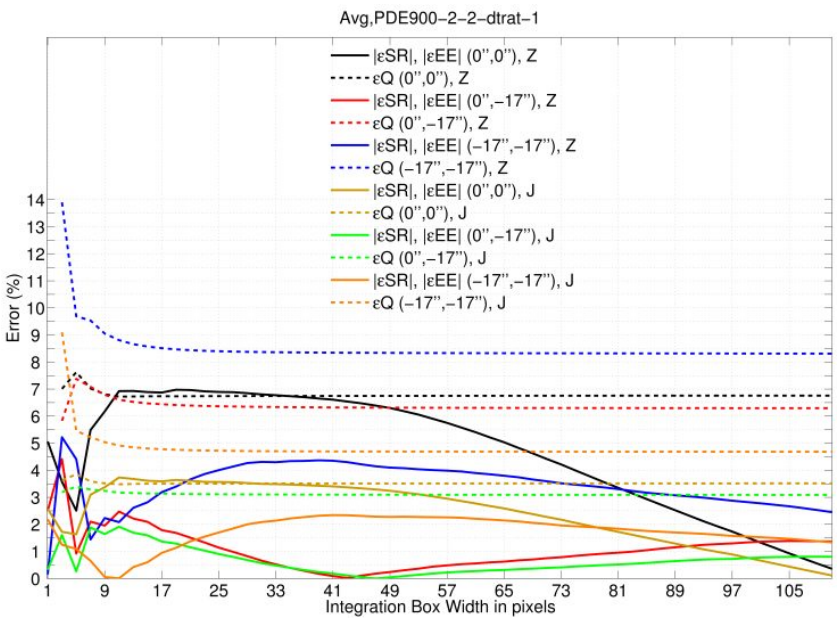

Figure 4: EE and PSF profile error versus window size. 
Finally, Figure 6 displays simulated and estimated PSFs as well as the estimation errors. Note (i) the $3 \times 3$ ghosts around the peak of the reconstructed PSFs and (ii) the darker zone inside the control radius of the reconstructed PSFs. Further analysis is required to correct those reconstruction artifacts. Figure 7 displays PSF X-cross-sections.
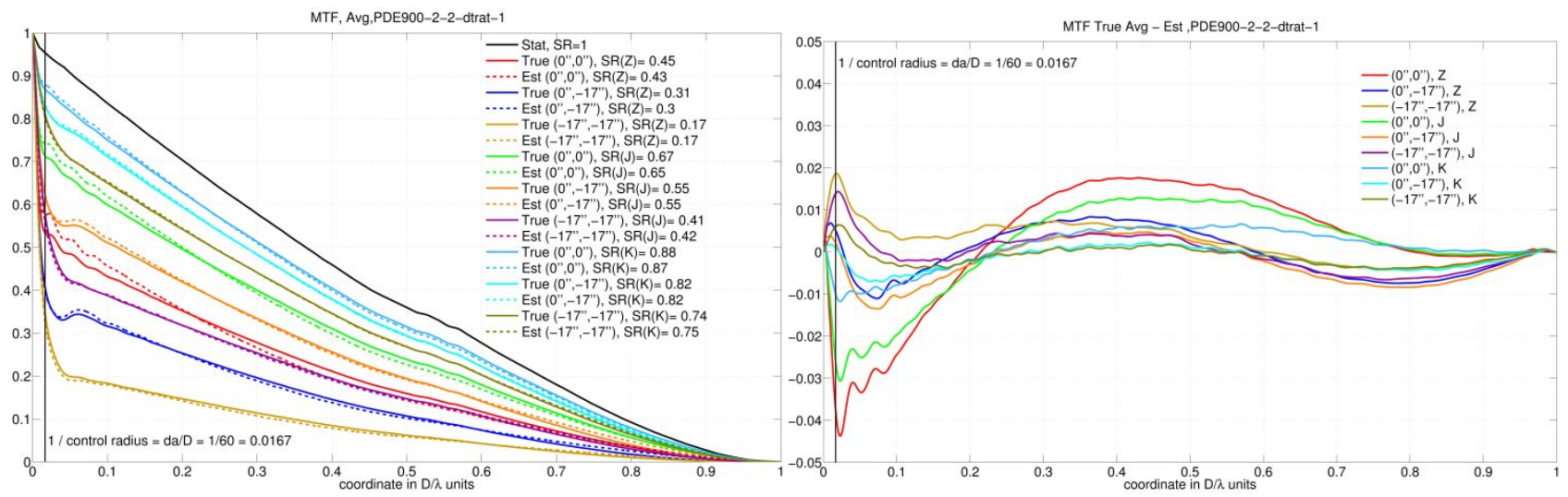

Figure 5: Left: J-band MTF x-cross-sections. Right: J-band MTF estimation error x-cross-sections.
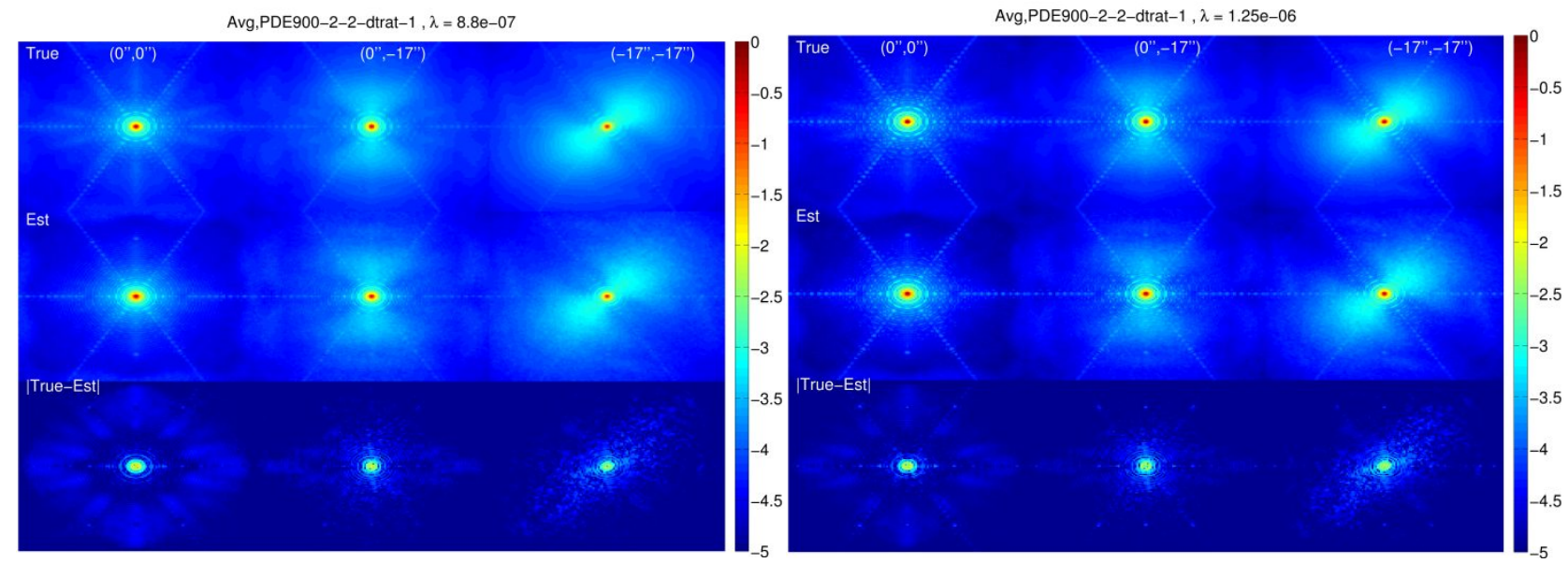

Figure 6: Top row are simulated PSFs ( $\log 10$ scale), middle row are estimated PSFs, bottom row are estimation errors. Left: Z-band, Right: J-band.
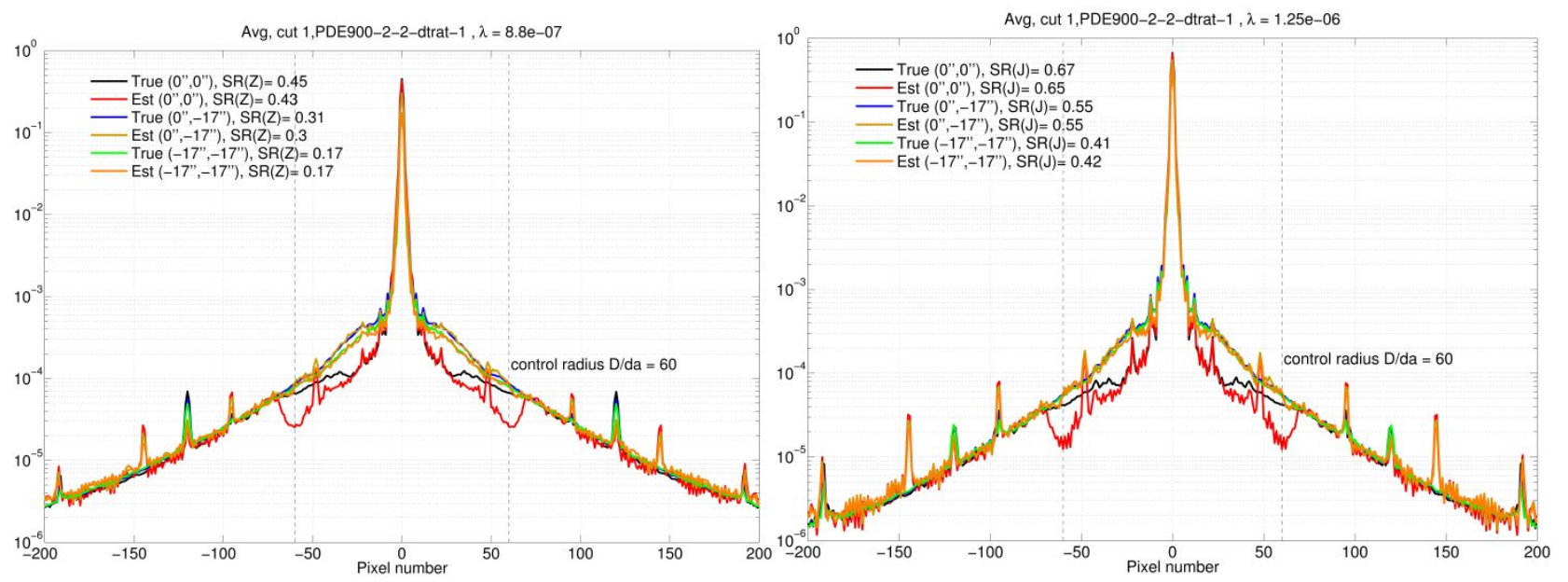

Figure 7: Simulated and estimated PSF X-cross-sections (log10 scale). Left: Z-band, Right: J-band. 


\section{CONCLUSIONS}

In this paper, PSFR simulation results for LGS MCAO on a 30m-class telescope were discussed. The PSFR algorithm uses pre-computed models for generalized fitting and aliasing, supplemented by the actuator error covariance matrix and the measurement noise covariance matrix for each WFS as telemetry data as described in [1]. For median Mauna Kea turbulence conditions ( 0.55 arcsec seeing) and for the expected NFRIAOS WFS noise, the actuator error based PSFR algorithm leads to Strehl Ratio (SR), enclosed energy (EE) and PSF profile errors below 8\% in Z-band, 5\% in J-band and $2 \%$ in $\mathrm{K}$-band assuming perfect knowledge of the turbulence profile and measurement noise. Further algorithm optimization could in principle reduce this residual error level.

\section{ACKNOWLEDGEMENTS}

The authors gratefully acknowledge the support of the TMT collaborating institutions. They are the Association of Canadian Universities for Research in Astronomy (ACURA), the California Institute of Technology, the University of California, the National Astronomical Observatory of Japan, the National Astronomical Observatories of China and their consortium partners, and the Department of Science and Technology of India and their supported institutes. This work was supported as well by the Gordon and Betty Moore Foundation, the Canada Foundation for Innovation, the Ontario Ministry of Research and Innovation, the National Research Council of Canada, the Natural Sciences and Engineering Research Council of Canada, the British Columbia Knowledge Development Fund, the Association of Universities for Research in Astronomy (AURA), the U.S. National Science Foundation and the National Institutes of Natural Sciences of Japan.

\section{REFERENCES}

[1] L.Gilles, L.Wang, M.Etsuko, J.P.Véran and C.Boyer "Point spread function analysis, modeling, simulations and laboratory testing for TMT NFIRAOS", Proc. AO4ELT5 (2017), doi:10.26698/AO4ELT5.0048

[2] C.Béchet, B.Ayancan, A.Guesalaga, C.Correia, B.Neichel, E.Masciadri and R.Conan "PSF reconstruction via full turbulence characterization end-to-end simulations", Proc. AO4ELT5 (2017), doi:10.26698/AO4ELT5.0051

[3] A.Ciurlo, T.Do, G.Witzel, J.Lu, M.Fitzgerald and A.Ghez, "PSF reconstruction for integral field spectrograph OSIRIS at Keck", Proc. AO4ELT5 (2017), doi:10.26698/AO4ELT5.0107

[4] R.Wagner, "PSF reconstruction and blind deconvolution for extremely large telescopes", Durham PSFR workshop (2018).

[5] M.Schoeck and L.Gilles, “TMT PSF Reconstruction Status”, Durham PSFR workshop (2018).

[6] E.Por, "PSF reconstruction: phase sorting interferometry", Durham PSFR workshop (2018).

[7] F.Cantalloube, "PSF reconstruction: dealing with the turbulence residual phase signature for high contrast imaging", Durham PSFR workshop (2018).

[8] O.Beltramo-Martin, "Improving photometry and astrometry in crowded fields using PSF reconstruction", Durham PSFR workshop (2018).

[9] C.Boyer, “Adaptive optics program update at TMT", Proc. AO4ELT5 (107), doi:10.26698/AO4ELT5.0131

[10] L.Gilles and B.Ellerebroek, "Real-time turbulence profiling with a pair of laser guide star Shack-Hartmann wavefront sensors for wide field adaptive optics systems on large to extremely large telescopes", J.Opt.Soc.Am. A27, A76-A83 (2010).

[11]L.Wang, MAOS, GitHub.

[12]L.Wang and B.Ellerbroek, "Computer simulations and real-time control of ELT AO systems using graphical processing units", Proc. SPIE 8447 (2012), doi: 10.1117/12.926500

[13] L.Gilles and B.Ellerbroek, "Split atmospheric tomography using laser and natural guide stars", J.Opt.Soc.Am.A25, 2427-2435 (2008). 